\title{
Individuals with moderately raised liver fat show a greater increase in liver fat in response to a high sugar diet
}

\author{
A. Ahmad ${ }^{1}$, C. Isherwood ${ }^{1}$, B. A. Fielding ${ }^{1}$, J. D. Bell ${ }^{2}$, E. L. Thomas ${ }^{2}$, G. Frost ${ }^{3}$, \\ F. Shojaee-Moradie ${ }^{1}$, M. Umpleby ${ }^{1}$ and B. A. Griffin ${ }^{1}$ \\ ${ }^{1}$ Faculty of Health \& Medical Sciences, University of Surrey, Guildford, Surrey GU2 7XH, ${ }^{2}$ Robert Steiner, MR Unit, \\ MRC Clinical Sciences Centre, Imperial College, London W12 OHS, ${ }^{3}$ Department of Medicine, Imperial College, \\ London SW7 2AZ, UK
}

Liver fat has been associated with the development of cardio-metabolic risk, and linked to a high intake of dietary sugars ${ }^{(1,2)}$. To investigate these associations further, we examined the effects of two, 12 week diets, high and low in sugars, on the percentage of liver fat, and the influence of the initial percentage of liver fat. The dietary intervention used a randomised, cross-over design, and was conducted in a free-living setting with men (aged 40-65, $n=17$ ) at increased cardio-metabolic risk according to the previously described 'RISCK' Study protocol ${ }^{(3)}$. Percentage liver fat was measured before and after each diet by magnetic resonance spectroscopy. The high and low sugar diets, that delivered $27 \%$ and $9 \%$ energy as sugar, with starch to sugar ratios of $1: 1$ and $3: 1$, were accompanied by a consistent increase and decrease in liver fat, respectively. These changes resulted in a significant difference in the percentage of liver fat between the two diets (post-diet medians: $7.3 \%$ versus $2.2 \%, p<0.05$ ). Individuals with a moderate increase in their percentage of liver fat at baseline $(>4.2 \%)$, showed a significantly greater increase in liver fat after the high sugar diet relative to the low sugar diet.

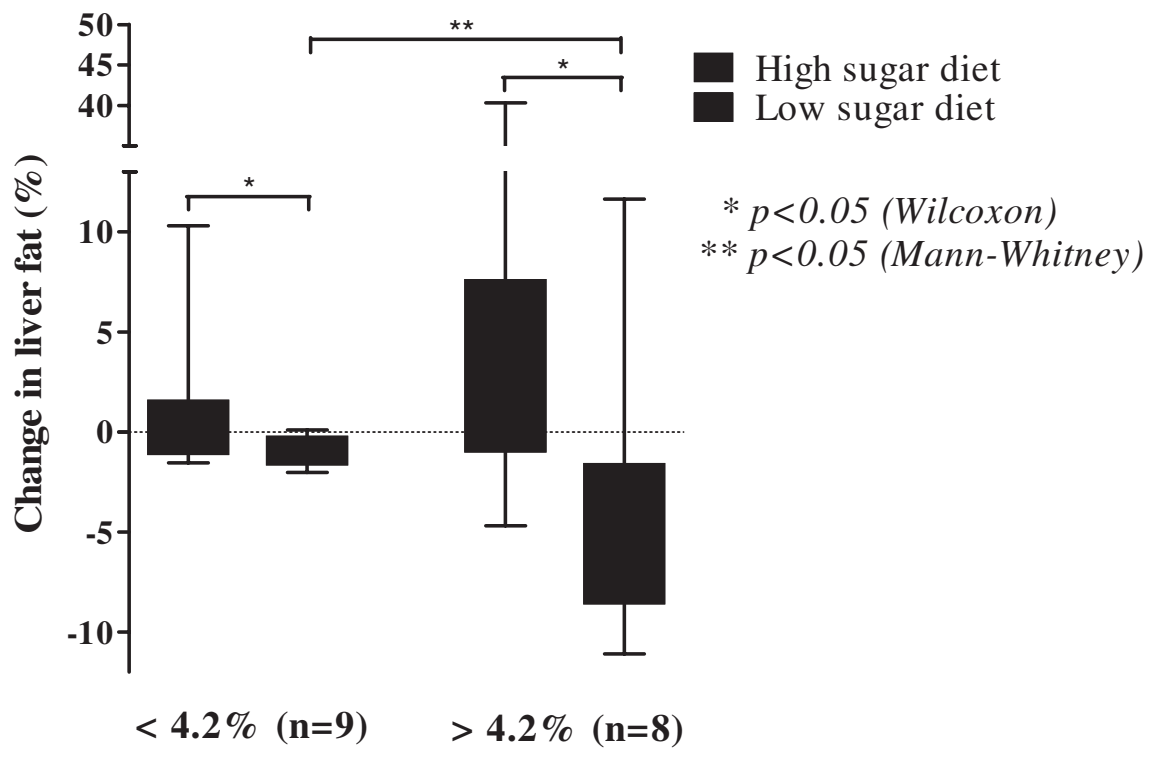

Liver fat group

In conclusion, these data confirm that a high sugar diet can promote an increase in liver fat. Moreover, this response was shown to be increased further in individuals with only a moderate elevation of liver fat, a finding that may have implications for the development of cardio-metabolic risk in this prevalent group.

This study was supported by the BBSRC (Grant No. BB/G009899/1)

1. Zelber-Sagi S, et al. (2007) J Hepatology 47, 711-717.

2. Stanhope KL \& PJ Havel (2008) Am J Clin Nutr 88, 1733S-1737S.

3. Jebb SA, et al. (2010) Am J Clin Nutr 92, 748-758. 